\title{
Suppression of Salient Objects Prevents Distraction in Visual Search
}

\author{
John M. Gaspar and John J. McDonald \\ Department of Psychology, Simon Fraser University, Burnaby, British Columbia, V5A 1S6 Canada
}

To find objects of interest in a cluttered and continually changing visual environment, humans must often ignore salient stimuli that are not currently relevant to the task at hand. Recent neuroimaging results indicate that the ability to prevent salience-driven distraction depends on the current level of attentional control activity in frontal cortex, but the specific mechanism by which this control activity prevents salience-driven distraction is still poorly understood. Here, we asked whether salience-driven distraction is prevented by suppressing salient distractors or by preferentially up-weighting the relevant visual dimension. We found that salient distractors were suppressed even when they resided in the same feature dimension as the target (that is, when dimensional weighting was not a viable selection strategy). Our neurophysiological measure of suppression - the $P_{D}$ component of the event-related potential—was associated with variations in the amount of time it took to perform the search task: distractors triggered the $P_{D}$ on fast-response trials, but on slow-response trials they triggered activity associated with working memory representation instead. These results demonstrate that during search salience-driven distraction is mitigated by a suppressive mechanism that reduces the salience of potentially distracting visual objects.

Key words: attention; distraction; EEG; ERP; suppression; visual search

\section{Introduction}

Converging evidence indicates that observers can ignore salientbut-irrelevant objects to attend to less salient visual-search targets in a timely fashion. Event-related potential (ERP) studies have revealed that salient distractors fail to elicit ERP activity associated with attentional selection when humans search repeatedly for the same target (Sawaki and Luck, 2010; Wykowska and Schübo, 2010, 2011; Kiss et al., 2012; Töllner et al., 2012; Jannati et al., 2013). Single-cell studies have revealed that monkeys can avoid making saccades to salient distractors when looking for a less-salient target and that the distractor triggers less activity on a parietal-lobe salience map when the monkey manages to do so (Ipata et al., 2006). Recent neuroimaging findings have highlighted the influence of frontal cortex in mitigating saliencedriven distraction: stronger pretrial activity in middle frontal gyrus leads to less distractor interference on an impending visualsearch task (Leber, 2010). Such results underscore the importance of top-down control of attention in search, but the neurocognitive mechanism that prevents the salience-driven misallocation of attention remains poorly understood.

Received Sept. 27, 2013; revised Feb. 20, 2014; accepted March 16, 2014.

Author contributions: J.M.G. and J.J.M. designed research; J.M.G. performed research; J.M.G. analyzed data; J.M.G. and J.J.M. wrote the paper.

This study was supported by Natural Sciences and Research Council of Canada, Canadian Foundation for Innovation, and the Canada Research Chairs program. We thank Ashley Livingstone and Christina Hull for assistance in collecting the data.

The authors declare no competing financial interests.

Correspondence should be addressed to either John Gaspar or John McDonald, Department of Psychology, Simon Fraser University, 8888 University Drive, Burnaby, BC, Canada V5A 156. E-mail: jmg2@sfu.ca or jmcd@sfu.ca.

DOI:10.1523/JNEUROSCI.4161-13.2014

Copyright $\odot 2014$ the authors $\quad 0270-6474 / 14 / 345658-09 \$ 15.00 / 0$
We aimed to determine the mechanism by which observers prevent salience-driven distraction in visual search. Two hypothetical mechanisms have been considered previously: salientsignal suppression and dimensional weighting. According to the salient-signal-suppression hypothesis, observers suppress signals arising from salient-but-irrelevant items when searching for a known target (Caputo and Guerra, 1998; Kumada and Humphreys, 2002; Sawaki and Luck, 2010; Jannati et al., 2013). According to the dimensional-weighting hypothesis, salience signals associated with the local contrast of visual stimuli are adjusted pre-attentively depending on the currently relevant visual feature dimension (Found and Müller, 1996). By this latter account, up-weighting of a relevant feature dimension (e.g., form) boosts the priority of stimuli defined by this dimension relative to the priority of stimuli defined by task-irrelevant dimensions (e.g., color), thereby facilitating the selection of targets in the presence of cross-dimension competition (Müller et al., 2009).

To evaluate these hypotheses, we recorded ERPs in search experiments involving cross- or within-dimension competition from salient distractors. We isolated two components-the N2pc and $\mathrm{P}_{\mathrm{D}}$ - to determine how distractors are processed. Whereas the N2pc is elicited by attended items and is hypothesized to reflect attentional selection (Luck and Hillyard, 1994a,b), the $P_{D}$ is elicited by unattended objects and is hypothesized to reflect attentional suppression (Hickey et al., 2009; Sawaki et al., 2012). In Experiment 1, participants searched for a color-singleton target and attempted to ignore a more salient color-singleton distractor (within-dimension competition). Critically, dimensional weighting would boost the salience of both singletons in this situation, leaving the distractor with highest priority for selection. Consequently, if no other mechanism were available to prevent 
salience-driven distraction, attention would be misallocated to the more physically salient distractor, resulting in a distractor $\mathrm{N} 2 \mathrm{pc}$. On the other hand, if suppression were able to prevent this attentional misallocation, the within-dimension distractor would elicit a $\mathrm{P}_{\mathrm{D}}$. The results revealed that a distractorsuppression mechanism helps to resolve the competition for attention during visual search, even when the target and distractor reside in the same feature dimension.

\section{Materials and Methods}

We conducted three experiments to determine the means by which observers deal with distraction in visual search. In Experiments 1 and 2, we tracked the processing of a salient color singleton while observers searched for either a less-salient shape singleton (cross-dimension competition) or a less-salient color singleton (within-dimension competition). Experiment 3 was identical to Experiment 1, but the target and distractor stimuli were reversed so that the distractor was less salient than the target.

Participants. Fifty-six neurologically typical students from Simon Fraser University were recruited from an undergraduate research pool and gave their informed consent to participate for pay or course credit. Eight subjects were excluded due to excessive ocular artifacts in the electro-oculogram (EOG). Of the remaining 48 participants, 16 participated in Experiment 1 ( 8 women, age $21.25 \pm 2.96$ years; 1 left-handed), 16 participated in Experiment 2 ( 10 women, age $22.37 \pm 3.82$ years; 0 left-handed), and 16 participated in Experiment 3 (11 women, age $20.14 \pm 2.42$ years; 1 left-handed). All subjects reported normal or normal-to-corrected visual acuity and were tested for typical color vision using Ishihara color test plates. The Research Ethics Board at Simon Fraser University approved all experimental protocol used in this study.

Stimuli and apparatus. Stimuli were presented on a 19 inch CRT monitor viewed at a distance of $57 \mathrm{~cm}$. In Experiment 1, the visual search array was comprised of 10 unfilled circles presented equidistant $\left(9.2^{\circ}\right)$ from a central fixation point onto a uniform black background $\left(0.5 \mathrm{~cd} / \mathrm{m}^{2}\right)$. Each circle was $3.4^{\circ}$ in diameter with a $0.3^{\circ}$ thick outline. On each trial, eight or nine of these circles were green $\left(11 \pm 0.1 \mathrm{~cd} / \mathrm{m}^{2}, x=0.275, y=\right.$ $0.599)$. The target and distractor were color singletons: the target was dark yellow $\left(11.1 \pm 0.1 \mathrm{~cd} / \mathrm{m}^{2}, x=0.384, y=0.528\right)$ and the distractor was red $\left(11 \pm 0.1 \mathrm{~cd} / \mathrm{m}^{2}, x=0.599, y=0.357\right)$. A vertical or horizontal gray line (gray: $11.1 \pm 0.1 \mathrm{~cd} / \mathrm{m}^{2}, x=0.295, y=0.361$ ) was contained within each of the circles. Experiment 2 was identical, except the target color singleton was replaced with a target shape singleton: an unfilled diamond $\left(4.2 \times 4.2^{\circ}\right)$ with a $0.3^{\circ}$ green $\left(11 \pm 0.1 \mathrm{~cd} / \mathrm{m}^{2}, x=0.275, y=\right.$ $0.599)$ outline. Experiment 3 was identical to Experiment 1, except the target was red and the distractor was dark yellow.

In the two within-dimension experiments (Experiments 1 and 3), salience was defined in terms of the local contrast between the green nontargets and each color singleton: the distance in chromaticity space between the red singleton and the green nontargets was greater than the distance between the yellow singleton and the green nontargets.

In each experiment, displays contained both target and distractor singletons on $50 \%$ of the trials (distractor-present trials). On the remaining $50 \%$ of trials, the target was the only singleton in the array (distractorabsent trials; Fig. 1). Target and distractor locations were varied to produce the following display configurations: lateral target, no distractor (33\%); midline target, no distractor (17\%); lateral target, midline distractor (11\%); lateral target, ipsilateral distractor (11\%); lateral target, contralateral distractor $(11 \%)$; midline target, lateral distractor $(11 \%)$; midline target, midline distractor $(6 \%)$. The order of these display configurations was determined pseudorandomly within each block of trials.

Procedure. At the beginning of each trial a fixation point appeared at the center of the screen for $800-1200 \mathrm{~ms}$. After this intertrial interval, one of the search displays described above was presented around the fixation point. Participants were instructed to maintain fixation on the central point and to identify the orientation of the gray line inside the target singleton by pressing one of two response buttons as quickly as possible. The search array remained visible for $100 \mathrm{~ms}$ after a response was registered, at which point the next trial began. Each experimental block was

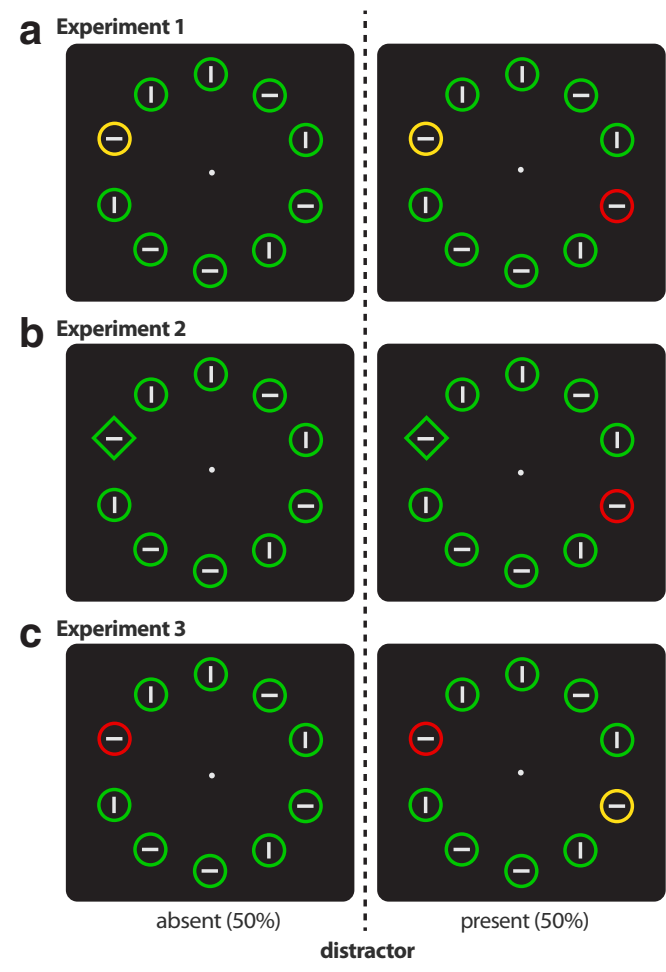

Figure 1. Example stimulus displays from the three experiments: (a) Experiment 1, (b) Experiment 2, (c) Experiment 3.

comprised of 36 trials, after which a mandatory minimum $5 \mathrm{~s}$ break was given to participants. Participants were encouraged to rest and to start the next block of trials when ready. The experiment contained 35 blocks for a total of 1260 trials per participant. At least 36 practice trials were given to each participant before commencing the experiment. Eye fixation was assessed on a trial-by-trial basis with EOG recordings (see details below).

Behavioral analysis. We assessed distractor interference in three steps. First, we compared reaction times (RTs; and error rates) obtained on distractor-present and distractor-absent trials to look at the overall level of interference. Second, we re-averaged RTs to lateral-target displays according to the number of positions between target and distractor. The distance ranged from 1 (d1; adjacent) to 5 ( $\mathrm{d} 5$; four intervening nontargets). A repeated-measures ANOVA with one five-level factor (targetdistractor distance; d1-d5) was used to assess search performance as a function of target-distractor proximity. Third, the RT obtained with target-distractor distance 5 (d5) was compared with the RT obtained on distractor-absent trials to determine whether the most distant distractors interfered with search performance.

Electrophysiological recording and analysis. ERPs were recorded from active sintered $\mathrm{Ag}-\mathrm{AgCl}$ electrodes from 125 standard sites and three nonstandard sites inferior to the standard occipital locations. Horizontal EOGs were recorded using two electrodes positioned $1 \mathrm{~cm}$ lateral to the external canthi and vertical EOGs were recorded using two electrodes positioned above and below the right eye. All EEG and EOG signals were digitized at $512 \mathrm{~Hz}$ and referenced in real time to an active commonmode electrode. Electrode offsets were monitored to ensure the quality of the data.

EEG processing and ERP averaging were performed using EventRelated Potential Software System (University of California, San Diego, CA). A semi-automated procedure was used to discard epochs of EEG contaminated by blinks, eye movements, or excessive noise (Green et al., 2008). Any trial with an artifact within a $1 \mathrm{~s}$ interval $(-200$ to $800 \mathrm{~ms}$ poststimulus) was rejected. Artifact-free epochs associated with the various display configurations of interest were then averaged separately to create ERP waveforms. The resulting ERPs were digitally low-pass filtered $(-3 \mathrm{~dB}$ point at $28 \mathrm{~Hz})$ and digitally re-referenced to the average of 
the left and right mastoids. All ERP amplitudes and baselines were computed using a $200 \mathrm{~ms}$ prestimulus window. The averaged event-related horizontal EOGs did not exceed $2 \mu \mathrm{V}$ for any individual participant, indicating their gaze remained within $0.3^{\circ}$ of the fixation point for a majority of the trials (cf. McDonald and Ward, 1999).

The primary analysis focused on ERPs elicited by the following display configurations: (1) lateral target, contralateral distractor; (2) lateral target, midline distractor; (3) lateral target, ipsilateral distractor; (4) midline target, lateral distractor; (5) lateral target, no distractor. Displays containing a lateral singleton and a midline singleton (configurations 2 and 4, above) enable isolation of lateralized ERP components (such as N2pc and $\mathrm{P}_{\mathrm{D}}$ ) because midline singletons do not trigger lateralizations (Woodman and Luck, 2003; Hickey et al., 2009). Displays containing ipsilateral and contralateral distractors (configurations 2 and 3 , above) enable investigation of gross target-distractor distance effects. Finally, distractorabsent displays with lateral targets (configuration 5, above) enable investigation of target processing in the absence of the distractor.

For each participant, ERPs to the various search displays were collapsed across left and right visual hemifields and left and right electrodes to produce waveforms recorded ipsilateral and contralateral to a lateral singleton (the target, unless otherwise noted). Lateralized ERP difference waveforms were then derived for each condition by subtracting the ipsilateral waveform from the corresponding contralateral waveform using lateral occipital electrode sites A10 and B7 (BioSemi nomenclature), which correspond to P07 and P08 of the international 10-10 electrode placement system. Negative voltages were plotted upward so that the $\mathrm{N} 2 \mathrm{pc}$ and $\mathrm{P}_{\mathrm{D}}$ would appear in these difference waveforms as upward and downward deflections, respectively. Mean amplitudes of the N2pc and $\mathrm{P}_{\mathrm{D}}$ were computed in a $40 \mathrm{~ms}$ interval centered on the peak N2pc time point (Experiments 1 and 2: 250-290 ms; Experiment 3: 220-260 ms), relative to a $200 \mathrm{~ms}$ prestimulus interval. Latency onsets were defined as the 70\%-of-peak-amplitude voltage in the $75-325 \mathrm{~ms}$ interval. Statistical tests were performed on jackknife-averaged ERPs and statistical thresholds were adjusted accordingly (Ulrich and Miller, 2001).

\section{Results}

\section{Experiment 1: distractor suppression resolves within-} dimension competition

\section{Behavior}

Overall, the salient distractor delayed search significantly in this within-dimension competition task. The mean RTs for distractorpresent trials were statistically longer than for distractor-absent trials ( 626 and $608 \mathrm{~ms}$, respectively; $t_{(15)}=5.40, p<0.001$ ). Participants also made more errors on distractor-present trials $(11.5 \%)$ than on distractor-absent $(2.8 \%)$ trials $\left(t_{(15)}=7.86, p<0.0001\right)$.

Previously, such behavioral results have been taken to indicate that observers misallocate attention to the location of the distractor before finding the target (Theeuwes, 1992). However, the overall distractor-interference effect has also been considered to reflect a nonspatial filtering cost (Folk and Remington, 1998). Some researchers have argued against a nonspatial filtering cost by showing that the magnitude of distractor interference depends on the spatial separation between target and distractor (Mounts, 2000a; Hickey and Theeuwes, 2011). In those studies, nearby distractors delayed search more than distant distractors. Although this spatial effect does not imply immediately that observers attended to the distractor, it is usually interpreted in the context of the original salience-driven-misallocation-of-attention hypothesis.

Here, we found that the magnitude of RT interference depended on target-distractor distance. As illustrated in Figure $2 a$, RTs decreased monotonically as the target-distractor distance was increased from distance 1 ( $\mathrm{d} 1$; adjacent) to distance 5 ( $\mathrm{d} 5$; four intervening items). This distance effect was statistically significant $\left(F_{(4,60)}=21.45, p<0.0001\right)$, and so a completely nonspatial filtering cost can be ruled out. One aspect of this spatial
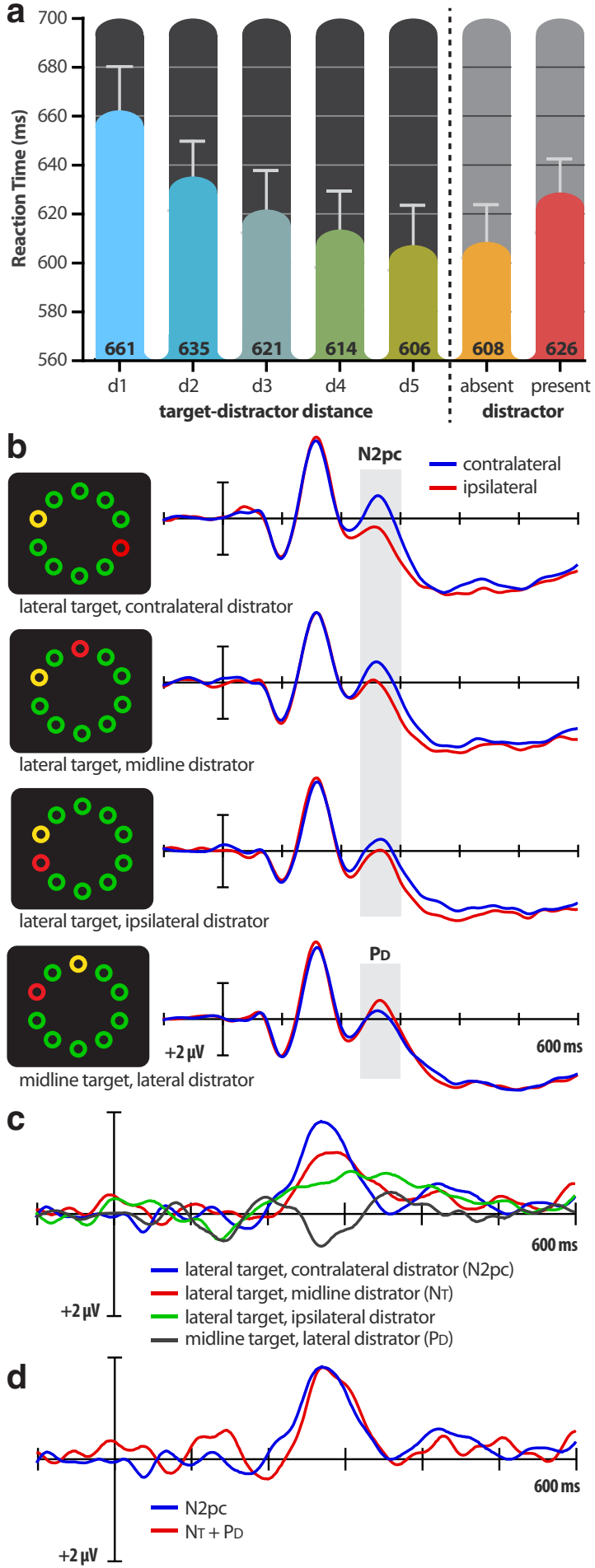

Figure 2. Main results from Experiment 1: (a) Mean response times (across participants; in milliseconds) for the five target-distractor distances $(\mathrm{d} 1-\mathrm{d} 5)$ and the overall distractor-absent and distractor-present trials. $\boldsymbol{b}$, Grand-average ERP waveforms for display configurations of interest, recorded at contralateral and ipsilateral occipital (P07/P08) electrode sites. c, Contralateral-minusipsilateral difference waveforms for the display configurations of interest. $\boldsymbol{d}$, Comparison of difference waveforms for the hypothetical N2pc (the absolute algebraic sum of the isolated $\mathrm{N}_{\mathrm{T}}$ and the isolated $P_{D}$ ) and the actual N2pc elicited by the lateral-target/contralateral-distractor display.

effect was inconsistent with the salience-driven-misallocationof-attention hypothesis. Specifically, the mean RT associated with the greatest target-distractor distance (distance 5) was not statistically different from the mean RT on distractor-absent tri- 
als (606 ms vs $608 \mathrm{~ms}$, respectively; $\left.t_{(15)}<1\right)$. That is, distant distractors did not delay search in the present search task.

\section{ERPs}

Figure $2 b$ shows ERP waveforms elicited by the four search display configurations of interest. The critical question in Experiment 1 was whether the lateral distractor would elicit an N2pc target was on the midline. The results revealed that the lateral distractor elicited not the N2pc but a $\mathrm{P}_{\mathrm{D}}$ component in this configuration. That is, the ERP waveform was more positive contralateral than ipsilateral to the distractor $\sim 250-300 \mathrm{~ms}$ after display onset. This can be seen clearly as a downward (positive) peak at $\sim 275 \mathrm{~ms}$ in the lateralized difference waveforms created by subtracting the ipsilateral ERPs from the corresponding contralateral ERPs (Fig. $2 c$ ). For each lateral-target configuration, the ERP was more negative contralateral to the target than ipsilateral to the target in the usual time range of the N2pc $(200-300 \mathrm{~ms}$; Fig. 2b,c). Based on the hypothesis that the $\mathrm{P}_{\mathrm{D}}$ reflects the operation of a suppressive mechanism that helps bias attention toward task-relevant objects (Hickey et al., 2009; Hilimire et al., 2012; Sawaki et al., 2012), these results indicate that observers suppressed the distractor and allocated attention to the target $\sim 250$ ms after display onset.

To assess the presence of lateralized ERP components in the $\mathrm{N} 2 \mathrm{pc}$ time interval, a pairwise $t$ test was used to compare the amplitudes of the contralateral and ipsilateral waveforms in the time range of the $\mathrm{N} 2 \mathrm{pc} / \mathrm{P}_{\mathrm{D}}$ for each display configuration. The mean $\mathrm{P}_{\mathrm{D}}$ amplitude for the midline-target, lateral-distractor display configuration was statistically significant in the $250-290 \mathrm{~ms}$ interval $\left(t_{(15)}=2.93\right.$, $p=0.01$ ). The mean $\mathrm{N} 2 \mathrm{pc}$ amplitudes for the lateral-target display configurations were all found to be statistically significant in the same interval (lateral target, contralateral distractor: $t_{(15)}=5.80, p<$ 0.001; lateral target, midline distractor: $t_{(15)}=4.81, p<0.001$; lateral target, ipsilateral distractor: $t_{(15)}=2.88, p=0.011$; lateral target, no distractor: $\left.t_{(15)}=8.47, p<0.001\right)$.

$N 2 p c=N_{T}+P_{D}$

Hickey et al. (2009) hypothesized that when the search display contains an attended target singleton and an unattended distractor singleton, the N2pc reflects two separable ERP components: an ERP negativity contralateral to the target (termed the target negativity, $\mathrm{N}_{\mathrm{T}}$ ), and the $\mathrm{P}_{\mathrm{D}}$ contralateral to the distractor. The present experiment offers the first evidence for this hypothesis.

As can be seen in Figure $2 c$, the target N2pc was largest when the distractor was on the opposite side of fixation (mean amplitude in 250-290 ms window: $-1.72 \mu \mathrm{V}$ ), smallest when the distractor was on the same side of fixation $(-0.61 \mu \mathrm{V})$, and intermediate when the distractor was on the vertical midline $\left(-1.12 \mu \mathrm{V} ; F_{(2,30)}=16.01, p=<0.001\right)$. This last display is critical for isolating the $\mathrm{N}_{\mathrm{T}}$ because the midline distractor cannot contribute to the lateralized ERP componentry. According to the summation hypothesis outlined above, the N2pc waves associated with the other two lateral-target displays vary around this baseline $\mathrm{N}_{\mathrm{T}}$ because of linear summation with the $\mathrm{P}_{\mathrm{D}}$ (isolated with the midline target, lateral distractor display). In other words, the $\mathrm{N}_{\mathrm{T}}$ and $\mathrm{P}_{\mathrm{D}}$ summate to increase the observed $\mathrm{N} 2 \mathrm{pc}$ amplitude when the target and distractor are on opposite sides of fixation and counteract each other to reduce the observed N2pc amplitude when the target and distractor are on the same side of fixation. This is exactly what we found. The target N2pc observed in the presence of a contralateral or ipsilateral distractor was, on average, $0.55 \mu \mathrm{V}$ larger or smaller than the $\mathrm{N}_{\mathrm{T}}$ observed in the presence of a midline distractor. The amplitude of the isolated $\mathrm{P}_{\mathrm{D}}$ $(0.50 \mu \mathrm{V})$ matched this amplitude difference very closely.
To address the additivity of $\mathrm{N}_{\mathrm{T}}$ and $\mathrm{P}_{\mathrm{D}}$ more directly, we created a $\mathrm{N}_{\mathrm{T}}+\mathrm{P}_{\mathrm{D}}$ waveform and compared it to the lateralized difference wave elicited by the lateral-target/contralateraldistractor display. The $\mathrm{N}_{\mathrm{T}}+\mathrm{P}_{\mathrm{D}}$ waveform was created in two steps: (1) the lateralized difference wave elicited by the midlinetarget/lateral-distractor display was inverted so as to make the polarity of the $\mathrm{P}_{\mathrm{D}}$ relative to the target when the target and distractor are on opposite sides of fixation, (2) this inverted $P_{D}$ waveform was added to the $\mathrm{N}_{\mathrm{T}}$ waveform (i.e., the lateralized difference wave to the lateral-target/midline-distractor display). The resulting $\mathrm{N}_{\mathrm{T}}+\mathrm{P}_{\mathrm{D}}$ waveform is displayed in Figure $2 d$ along with the lateralized difference waveform that was actually observed in response to the lateral target, contralateral distractor display. The waveforms contain remarkably similar negative peaks in the N2pc time range. The peak latency of the $\mathrm{N}_{\mathrm{T}}+\mathrm{P}_{\mathrm{D}}$ peak (252 ms) was statistically indistinguishable from the peak latency of the observed N2pc (246 ms; $\left.t_{(15)}=1.12, p=0.28\right)$. Likewise, the mean amplitudes of the two peaks did not differ statistically $\left(-1.62 \mu \mathrm{V}\right.$ vs $\left.-1.72 \mu \mathrm{V} ; t_{(15)}=0.36, p=0.73\right)$.

\section{Comparison of $P_{D}$ for fast- and slow-response trials}

The preceding ERP analyses demonstrated that attended targets and salient but to-be-ignored distractors trigger oppositepolarity ERP activity in the same time interval following a search display. Prior work has shown that the $\mathrm{P}_{\mathrm{D}}$ is often in evidence when selective processing of the eliciting stimulus is to be avoided (Hickey et al., 2009) or terminated (Sawaki et al., 2012), which has led researchers to hypothesize that the ERP positivity reflects active suppression of processing. In line with this hypothesis, the amplitude of distractor-elicited ERP activity in the $\mathrm{N} 2 \mathrm{pc} / \mathrm{P}_{\mathrm{D}}$ time range has been found to vary as a function of RT. In particular, distractors were found to elicit a $P_{D}$ when target-absent responses were made quickly and to elicit an N2pc when those responses were made more slowly (Sawaki et al., 2012). We performed a similar analysis of the ERPs elicited by the midline target, lateral distractor display. The trials for each participant were subdivided into fast-response and slow-response subsets, depending on whether the RT was shorter or longer than the median RT for that display configuration. If the distractor was suppressed to bias attention toward the target, then variations in the application of this suppression should influence the speed of targetdiscrimination responses. This was found to be the case. As illustrated in Figure 3, the $\mathrm{P}_{\mathrm{D}}$ was significantly larger for fast-response trials than for slow-response trials $\left(t_{(15)}=2.37, p=0.03\right)$. Follow-up analyses revealed two important results. First, the $\mathrm{P}_{\mathrm{D}}$ was not significant on slow-response trials $\left(t_{(15)}=0.87, p=\right.$ 0.40 ). Second, a contralateral negative ERP component was found to be significant in the 325-425 ms interval on slowresponse trials $\left(t_{(15)}=2.36, p=0.03\right)$, but not on fast-response trials $\left(t_{(15)}=0.17, p=0.87\right)$. This negativity likely reflects contralateral delay activity (CDA) that has been associated with active representation of items in visual short-term memory (even in visual-search tasks; Vogel and Machizawa, 2004; Jolicoeur et al., 2008). In compound-search tasks, this active representation might be required for the identification of the relevant stimulus features (e.g., orientation of the line inside the target shape; Mazza et al., 2007; Jannati et al., 2013; McDonald et al., 2013). Finally, a small distractor-elicited N2pc is visible $200-220 \mathrm{~ms}$ after display onset in the slow-response ERPs (Fig. 3), but this activity did not reach significance $\left(t_{(15)}=1.56, p=0.14\right)$. 


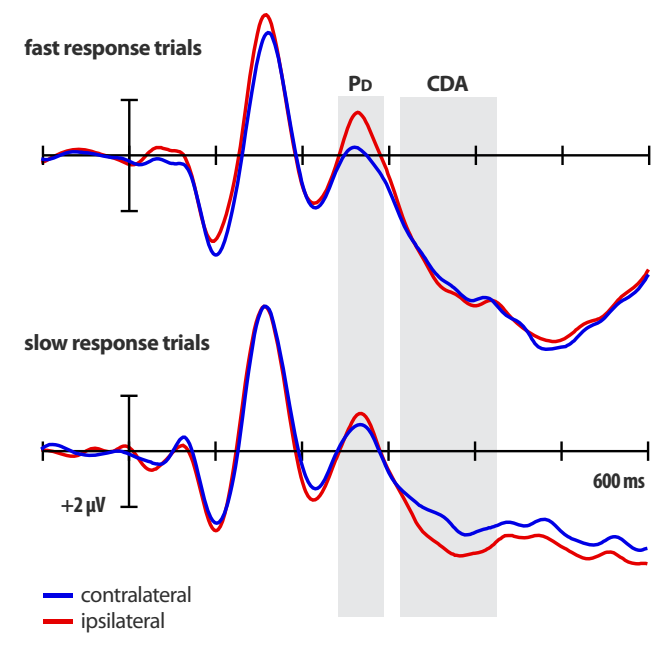

Figure 3. ERPs elicited by the midline target, lateral distractor display, averaged separately for fast-response and slow-response trials. The shaded boxes represent the time windows of the $P_{D}$ and subsequent $C D A$.

\section{Experiment 2: distractor suppression helps resolve cross-dimension competition}

Overview

The results of Experiment 1 show that a salient-but-irrelevant color singleton is suppressed when observers search for another, less salient color singleton. Experiment 2 was designed to determine whether the same suppression-based mechanism helps to prevent salience-driven misallocation of attention when the target and distractor singletons are defined on the basis of different feature dimensions. Experiment 2 was identical to Experiment 1, except participants searched for a green diamond instead of a yellow circle. This specific shape singleton was used because it is known to be less salient than high-contrast color singletons like the red singleton used here (Theeuwes, 1991, 1992).

Whereas dimensional weighting would not serve as a viable selection mechanism in Experiment 1, both hypothetical selection mechanisms could be used to reduce distraction in Experiment 2 because the target and distractor were defined along separate feature dimensions. Accordingly both the distractorsuppression hypothesis and the dimensional weighting hypothesis would predict reduced behavioral distraction in Experiment 2. If distraction were prevented entirely by distractor suppression then the distractor used in Experiment 1 should elicit a similar $P_{D}$ in Experiment 2. If, however, distraction were prevented entirely by dimensional up-weighting of the target, then there would be no reason to suppress the distractor and thus no $\mathrm{P}_{\mathrm{D}}$ would be expected to occur.

\section{Behavior}

As in Experiment 1, responses were significantly slower and more error prone on distractor-present trials (645 ms; 12.4\% errors) than on distractor-absent trials $\left(638 \mathrm{~ms} ; 3.2 \%\right.$ errors; RT: $t_{(15)}=$ 2.85, $p=0.012$; Error: $\left.t_{(15)}=7.57, p<0.0001\right)$. The delay, however, was significantly smaller than what was observed in Experiment 1 ( $7 \mathrm{~ms}$ vs $18 \mathrm{~ms} ; t_{(30)}=2.66, p=0.012$ ). Together, these results indicate that salient color-singleton distractors impair search for the shape-singleton target, but that less interference occurs when the target and distractor compete across dimensions rather than within the same dimension.

Following the distractor-interference analyses, median RTs were compared across the five possible target-distractor distances. Although the RTs did not drop monotonically as in Ex-
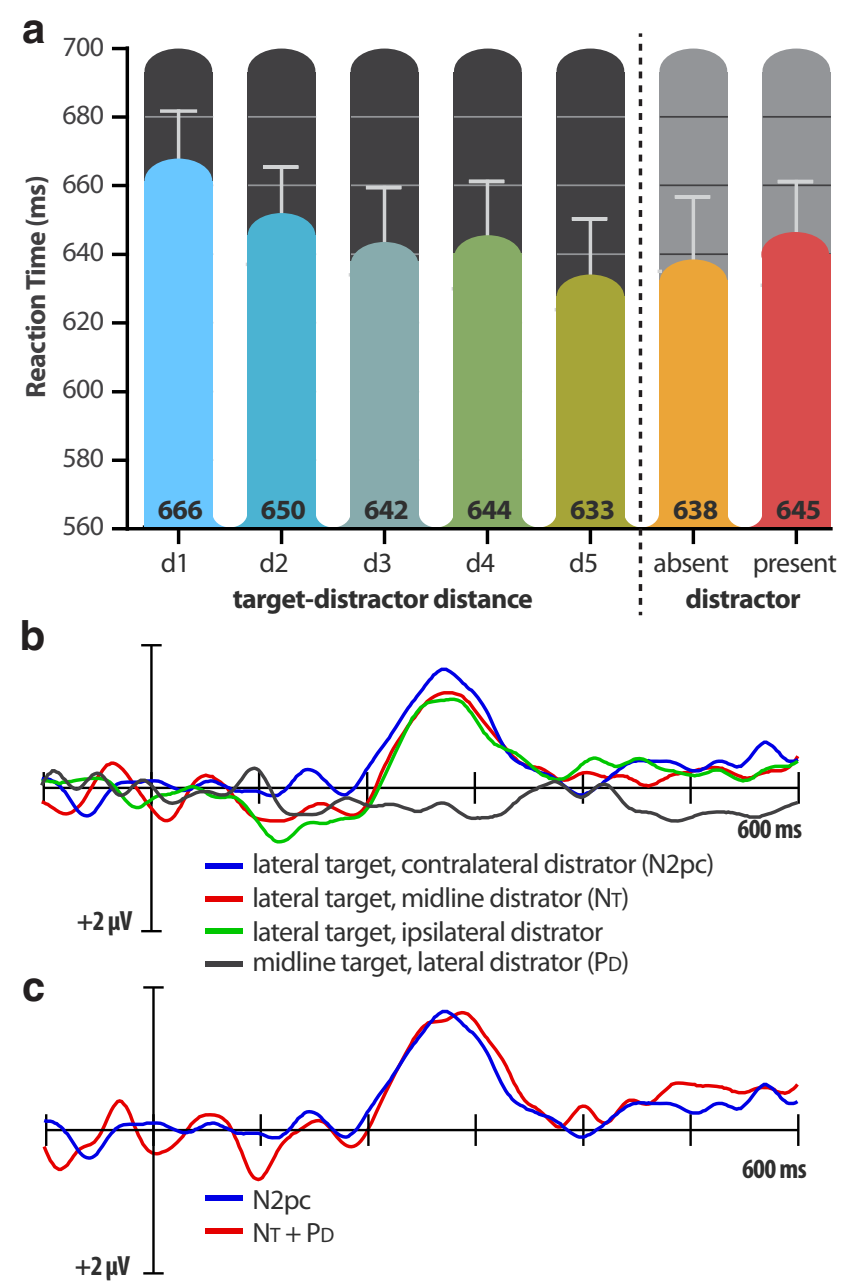

Figure 4. Results from Experiment 2: (a) Mean response times (across participants; in milliseconds) for the five target-distractor distances (d1-d5) and the overall distractor-absent and distractor-present trials. $\boldsymbol{b}$, Contralateral-minus-ipsilateral difference waveforms for the display configurations of interest, recorded at P07/P08 electrode sites. C, Comparison of difference waveforms for the hypothetical $\mathrm{N} 2 \mathrm{pc}$ (the absolute algebraic sum of the isolated $\mathrm{N}_{T}$ and the isolated $\mathrm{P}_{\mathrm{D}}$ ) and the actual N2pc elicited by the lateral-target/contralateral-distractor display.

periment 1 , nearby distractors were generally more disruptive than distant distractors, leading to a main effect of targetdistractor distance $\left(F_{(4,60)}=9.15, p<0.0001\right.$; Fig. $\left.4 a\right)$. Importantly, RTs for the longest target-distractor distance were not statistically different from RTs to distractor-absent displays $\left(t_{(15)}=1.06, p=0.27\right)$, showing that the most distant distractors did not delay search.

\section{ERPs}

Figure 5 displays the contralateral-ipsilateral difference waveforms for lateral-target displays on distractor-absent trials across all three experiments. The target N2pcs observed in Experiments 1 and 2 were nearly identical. Statistical analysis revealed that the onset latencies and amplitudes were statistically indistinguishable across the two experiments (latency: $t_{(30)}=0.24, p=0.82$; amplitude: $\left.t_{(30)}=0.69, p=0.49\right)$. These findings indicate that the salience of the form singleton in Experiment 2 was on par with the salience of the color-singleton target in Experiment 1.

Figure $4 b$ displays the contralateral-ipsilateral difference waveforms for the display configurations of interest. Unsurprisingly, displays containing lateral targets elicited the N2pc waves beginning $\sim 200$ after display onset. The mean amplitudes of 


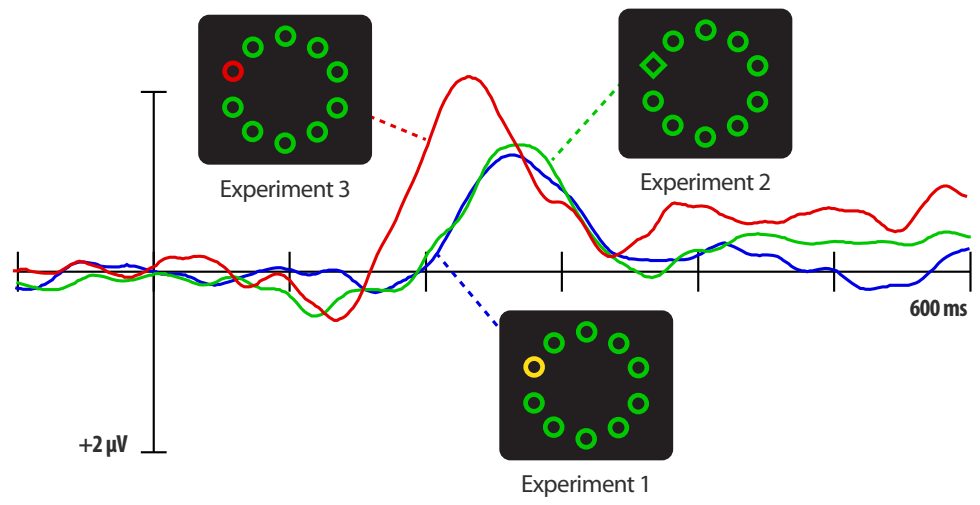

Figure 5. Grand-average ERP waveforms for lateral-target, no distractor displays in the three experiments, recorded at contralateral and ipsilateral occipital (P07/P08) electrode sites.

these N2pc waves were significant in the 250-290 ms interval $\left(t s_{(15)} \geq 4.16\right.$, ps $\left.\leq 0.001\right)$. Although there were small variations in N2pc amplitude across the three lateral-target configurations, these variations were only marginally significant $\left(F_{(2,30)}=2.50\right.$, $p=0.10)$.

Critically, there was a small but significant $\mathrm{P}_{\mathrm{D}}$ in response to the midline-target/lateral-distractor display $\left(t_{(15)}=2.40, p=\right.$ 0.03 ), indicating that participants suppressed the location of the salient distractor. This $\mathrm{P}_{\mathrm{D}}$ appeared to be smaller than the $\mathrm{P}_{\mathrm{D}}$ observed in Experiment 1, and the difference in $\mathrm{P}_{\mathrm{D}}$ amplitude across the two experiments was found to be significant by onetailed $t$ test, $t_{(30)}=1.766, p=0.044$. The use of a one-tailed test here is justified because we hypothesized at the outset that the $\mathrm{P}_{\mathrm{D}}$ may be absent entirely in Experiment 2 (Jannati et al., 2013).

As in Experiment 1, the isolated $\mathrm{N}_{\mathrm{T}}$ and $\mathrm{P}_{\mathrm{D}}$ waveforms obtained on the lateral target, midline distractor and the midline target, lateral distractor trials, respectively, summed to equal the $\mathrm{N} 2 \mathrm{pc}$ obtained on lateral target, contralateral distractor trials (Fig. 4c).

\section{Experiment 3: no suppression when the target is more salient than the distractor \\ Overview}

Despite the evidence linking the $\mathrm{P}_{\mathrm{D}}$ to suppression in Experiment 1 , it is possible that purely sensory processes contributed to the contralateral positivity in the present study. This seemed to be the case in one recent study, wherein a late contralateral positivity was linked to the mere appearance of a red color singleton (Fortier-Gauthier et al., 2013). We tested the alternative sensory explanation by reversing the task assignment of the two color singletons that were used in Experiment 1: participants searched for the salient red singleton and attempted to ignore the less salient yellow singleton. If the $\mathrm{P}_{\mathrm{D}}$ observed in Experiment 1 reflects exogenous processing of the salient red singleton, the red target should still elicit a $\mathrm{P}_{\mathrm{D}}$-like positivity (preceded by an N2pc, just as in Fortier-Gauthier et al., 2013). If, on the other hand, that $P_{D}$ reflected suppression, then neither the salient red singleton nor the less-salient yellow singleton should elicit the $\mathrm{P}_{\mathrm{D}}$ here because (1) the red singleton that elicited the $P_{D}$ in Experiment 1 is now task relevant and thus should not be ignored, and (2) the yellow singleton is already less salient than the target red singleton, making additional suppression unnecessary. The dimensionalweighting hypothesis did not provide a plausible account of the $\mathrm{P}_{\mathrm{D}}$ observed in Experiment 1 due to the within-dimension competition conditions, and so it will not be considered further here.

\section{Behavior}

Even though the distractor was less salient that the target, responses were still significantly slower on distractor-present trials ( $575 \mathrm{~ms} ; 9.7 \%$ errors) than on distractorabsent trials (568 ms; $2.7 \%$ errors; RT: $t_{(15)}=4.34, p=0.0005$; Error: $t_{(15)}=$ $5.61, p<0.0001)$. The delay was significantly smaller than the delay observed in Experiment 1 ( $7 \mathrm{~ms}$ vs $18 \mathrm{~ms} ; t_{(30)}=2.83$, $p=0.008$ ), but it was indistinguishable from the delay observed in Experiment 2 (7 ms each; $\left.t_{(30)}=0.153, p=0.88\right)$.

In Experiments 1 and 2, the median RTs generally decreased as the targetdistractor distance increased, indicating that nearby distractors were more disruptive than distant distractors. As can be seen in Figure $6 a$, this was not the case in Experiment $3\left(F_{(4,60)}=0.531, p=0.713\right)$. The absence of a targetdistractor distance effect indicates that distractor interference arises from nonspatial filtering when the distractor is less salient than the target.

\section{ERPs}

Figure 3 shows the contralateral-ipsilateral difference waves for the lateral target, no-distractor display, and Figure $6 b$ shows the contralateral-ipsilateral difference waves for the distractorpresent displays of interest. The N2pc was seen contralateral to the target for each of the lateral-target displays. Visual inspection of the ERPs revealed that the N2pc peaked earlier than in the previous two experiments, as was the case in Fortier-Gauthier et al. (2013). Consequently, we measured the N2pc in a $40 \mathrm{~ms}$ window centered on an earlier peak (220-260 ms). The lateral-target $\mathrm{N} 2 \mathrm{pc}$ waves were all significant in this interval $\left(t_{\mathrm{s}_{(15)}} \geq 3.188\right.$, $p s \leq 0.006$ ). To test whether the N2pc was significantly earlier than in Experiment 1, we created jackknife-averaged waveforms for the lateral-target/midline distractor display configuration of each experiment and compared the $70 \%$ fractional peak latencies of the N2pc waves by unpaired $t$ test (Ulrich and Miller, 2001). The results revealed that the N2pc to the red target $(207 \mathrm{~ms}$ ) was significantly earlier than the N2pc to the less-salient yellow target (249 $\left.\mathrm{ms} ; t_{(30)}=4.79, p=0.001\right)$. This finding is in line with previous claims that N2pc latency depends on stimulus salience (Töllner et al., 2011).

Although the red-target N2pc peaked relatively early in Experiment 3 , there was no indication that it was followed by a $\mathrm{P}_{\mathrm{D}}$-like positivity in the 250-290 ms time interval when the distractor was on the vertical meridian or when the distractor was absent (red waveforms in Figs. 6b, Fig. 3, respectively). Instead, the mean amplitude remained negative within this window for both conditions: $-1.17 \mu \mathrm{V}\left(t_{(15)}=2.051, p=0.058\right)$ and $-1.36 \mu \mathrm{V}\left(t_{(15)}=\right.$ $2.864, p=0.012$ ), respectively. These findings rule out the alternative explanation for the $\mathrm{P}_{\mathrm{D}}$, namely that some part of it reflects exogenous processing of a salient red color singleton. Importantly, there was also no $\mathrm{P}_{\mathrm{D}}$ elicited by the less-salient yellow distractor in Experiment $3\left(t_{(15)}=1.112, p=0.284\right)$. Together with the behavioral results (and in particular, the lack of a targetdistractor distance effect), these findings support two conclusions. First, the $\mathrm{P}_{\mathrm{D}}$ observed in the present study reflects suppression of a salient-but-irrelevant color singleton. Second, such suppression does not (necessarily) occur if a salient item is 


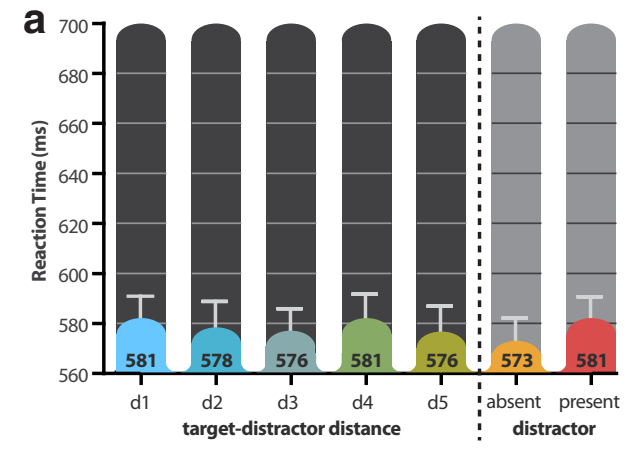

b

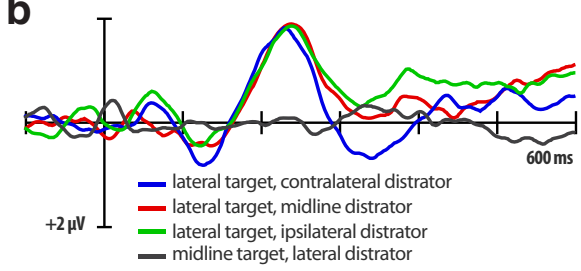

Figure 6. Results from Experiment 3: (a) Mean response times (across participants; in milliseconds) for the five target-distractor distances (d1-d5) and the overall distractor-absent and distractor-present trials. $\boldsymbol{b}$, Contralateral-minus-ipsilateral difference waveforms for the display configurations of interest, recorded at P07/P08 electrode sites.

task relevant or if an irrelevant item is less salient than the to-beattended item.

On first blush, this latter claim seems at odds with our lab's original $P_{D}$ study (Hickey et al., 2009), wherein a reasonably inconspicuous distractor elicited a $\mathrm{P}_{\mathrm{D}}$ when observers searched for a more salient target. However, in the original $\mathrm{P}_{\mathrm{D}}$ study, search displays contained only two items (target and distractor), whereas in the present study, search displays contained 10 items. Observers often attend to irrelevant items under low-perceptual load conditions (Lavie, 1995), and so it is likely that nonsalient distractors elicit the $\mathrm{P}_{\mathrm{D}}$ in such conditions to keep those items from accessing subsequent stages of processing.

\section{Discussion}

The ERP method has been helpful in addressing outstanding issues of visual search and attention capture. Using the N2pc, researchers have found that salient distractors are sometimes attended (Hickey et al., 2006; McDonald et al., 2013) and sometimes ignored (Wykowska and Schübo, 2010, 2011; Kiss et al., 2012; Töllner et al., 2012; Jannati et al., 2013). All of these prior studies involved cross-dimension competition, suggesting that the ability to ignore the distractor depends critically on a dimensional weighting mechanism. In the present study, however, a salient distractor elicited an electrophysiological index of suppression-the $\mathrm{P}_{\mathrm{D}}$ - even when the target and distractor were both color singletons, that is, when dimensional weighting would not enable selection of the less-salient target. Critically, the $\mathrm{P}_{\mathrm{D}}$ was in evidence on fast-response trials but not on slow-response trials in Experiment 1, suggesting that the suppression-based mechanism depends on fluctuations in attentional set. On slow-response trials, the distractor was found to elicit a CDA, which has been associated with the identification of the relevant stimulus features within working memory (Mazza et al., 2007). The absence of a distractor CDA on fast-response trials indicates that the distractor failed to gain access to working memory. Together these results suggest that the $\mathrm{P}_{\mathrm{D}}$ reflects the operation of a suppressive mechanism that minimizes the im- pact of the salient distractor on subsequent stages of processing associated with working memory.

The evidence for dimensional weighting was less clear. At first glance, it is tempting to attribute the $\mathrm{P}_{\mathrm{D}}$ observed in Experiment 2 (the cross-dimension search task) to the down-weighting of the distractor's task-irrelevant dimension. Such a conclusion would not be out of line with current views of dimensional weighting that emphasize suppression of the irrelevant distractor dimension over the up-weighting of the target dimension (Geyer et al., 2008; Müller et al., 2009; Töllner et al., 2012). However, we believe that it is more parsimonious to attribute this $\mathrm{P}_{\mathrm{D}}$ to locationbased suppression that is independent of dimensional weighting for two reasons. First, the $\mathrm{P}_{\mathrm{D}}$ components were similar across the within- and cross-dimension search task, suggesting that they were associated with the same selection mechanism. Because dimensional weighting was not a viable search strategy in Experiment 1 , such a common mechanism is unlikely to be related to dimensional weighting. Second, although similar, the $\mathrm{P}_{\mathrm{D}}$ was considerably larger in the within-dimension search task (Experiment 1) than in the cross-dimension search task (Experiment 2). We suspect that the $P_{D}$ was relatively small in Experiment 2 and in a previous cross-dimension search task (Jannati et al., 2013), because a dimensional weighting strategy increased the salience of the target and thus reduced the need to suppress the distractor.

Overall, the present results are consistent with a salient-signal suppression view of attentional control (Sawaki and Luck, 2010; Jannati et al., 2013). According to this perspective, (1) salient visual items compete for control (that is, they generate competing "attend to me" signals) during a stage of visual processing that precedes selection and (2) the visual system decides whether to select the location of the most salient item for further processing (selection for identification) or to selectively suppress that location to avoid further processing of the item there. This decision may be based on an observer's current attentional control settings (Folk et al., 1992; Sawaki and Luck, 2010), recent history of stimulus selection (Awh et al., 2012), or both.

The conclusion that salient distractors can be suppressed raises an interesting follow-up question: What causes the spatially modulated distractor interference effect? Given that the RT interference effect in Experiments 1 and 2 was largely determined by the target-distractor distance, alternative explanations based on entirely nonspatial filtering seem unlikely. Previously such distance effects were chalked up to a zone of inhibition surrounding the attended location that delayed redeployment of attention from an attended distractor to a nearby (inhibited) target (Mounts, 2000b; Hickey and Theeuwes, 2011). The absence of a target N2pc in Experiments 1 and 2 is inconsistent with this view. We propose that the RT interference is due to neural ambiguity that arises when an attended target and a suppressed distractor are within the same cellular receptive fields. By this account, suppression signals associated with the distractor and facilitatory signals associated with the target are in direct opposition, making it more difficult to identify or act upon the target stimulus. This proposal is generally consistent with the view that the N2pc reflects both target selection and distractor suppression (Hickey et al., 2009). It is also consistent with the ERP results from Experiment 1: the target N2pc was largest when the distractor was far away and smallest when the distractor was nearby.

This last finding may seem at odds with the results of Luck et al. (1997a), where the N2pc was found to be larger when the target was accompanied by a nearby distractor than when the target was the sole item in its visual field (Luck et al., 1997b). However, the additional distractors used in Luck et al. (1997a) 
were arguably less salient than the target (gray items vs colored item) and thus were unlikely to have triggered a $\mathrm{P}_{\mathrm{D}}$. Thus, in that study, the increase in target-elicited N2pc would not have been countered by an opposite-polarity component associated with the nearby distractor. In contrast, in the present study, the number of items near the target remained constant (no setsize manipulation) and the distractor was more salient than the target (in Experiments 1 and 2). Under these conditions, the $\mathrm{P}_{\mathrm{D}}$ appeared to counter the target-elicited $\mathrm{N} 2 \mathrm{pc}$ when the distractor and target were side-by-side. The amplitude of the target N2pc did not appear to vary, perhaps because set size was held constant.

The $\mathrm{P}_{\mathrm{D}}$ observed in the present study may be related to the cellular suppression observed in occipital, inferior temporal, and parietal areas of the monkey brain. Previous single-cell studies have shown that neurons in V4 and the inferior temporal (IT) cortex respond less vigorously to an otherwise effective stimulus (i.e., one that normally elicits a strong response from the cell) when the monkey covertly attends to an ineffective stimulus in the receptive field (Moran and Desimone, 1985). In saccade tasks, responses of IT neurons to effective distractors become suppressed $\sim 200 \mathrm{~ms}$ after display onset and 90-120 ms before the monkey saccades to an ineffective target (Chelazzi et al., 1993). Similar response suppression occurs in the lateral intraparietal (LIP) area when monkeys are trained to saccade to a shapesingleton target and to ignore a salient, known-in-advance, colorsingleton distractor (Ipata et al., 2006). In this case, neurons initially respond equally well to all stimuli, but by $\sim 90 \mathrm{~ms}$ after display onset, responses to the singleton distractor are significantly weaker than responses to the target and non-singleton distractors. This cellular response reduction was found to correlate with the animals' ability to ignore the singleton distractor on different days. It is likely that this early ( $\sim 90 \mathrm{~ms}$ poststimulus) suppression of LIP activity reflects reduction of distractor saliency on a master priority map, whereas the later $(\sim 200 \mathrm{~ms}$ poststimulus) suppression in IT reflects subsequent suppression of stimulus features at that location in object-processing regions of the ventral visual pathway. The timing of the $\mathrm{P}_{\mathrm{D}}$ highlighted in the present study is similar to the "late" single-unit suppression in IT. Thus, at present, we regard the $\mathrm{P}_{\mathrm{D}}$ to be a consequence of suppression in homologous object-processing regions of the human visual cortex.

\section{References}

Awh E, Belopolsky AV, Theeuwes J (2012) Top-down versus bottom-up attentional control: a failed theoretical dichotomy. Trends Cogn Sci 16: 437-443. CrossRef Medline

Caputo G, Guerra S (1998) Attentional selection by distractor suppression. Vision Res 38:669-689. CrossRef Medline

Chelazzi L, Miller EK, Duncan J, Desimone R (1993) A neural basis for visual search in inferior temporal cortex. Nature 363:345-347. CrossRef Medline

Folk CL, Remington R (1998) Selectivity in distraction by irrelevant featural singletons: evidence for two forms of attentional capture. J Exp Psychol Hum Percept Perform 24:847-858. CrossRef Medline

Folk CL, Remington RW, Johnston JC (1992) Involuntary covert orienting is contingent on attentional control settings. J Exp Psychol Hum Percept Perform 18:1030-1044. CrossRef Medline

Fortier-Gauthier U, Dell'acqua R, Jolicoeur P (2013) The "red-alert" effect in visual search: evidence from human electrophysiology. Psychophysiology 50:671-679. CrossRef Medline

Found A, Müller HJ (1996) Searching for unknown feature targets on more than one dimension: investigating a "dimension-weighting" account. Percept Psychophys 58:88-101. CrossRef Medline

Geyer T, Müller HJ, Krummenacher J (2008) Expectancies modulate atten- tional capture by salient color singletons. Vision Res 48:1315-1326. CrossRef Medline

Green JJ, Conder JA, McDonald JJ (2008) Lateralized frontal activity elicited by attention-directing visual and auditory cues. Psychophysiology 45: 579-587. CrossRef Medline

Hickey C, Theeuwes J (2011) Context and competition in the capture of visual attention. Atten Percept Psychophys 73:2053-2064. CrossRef Medline

Hickey C, McDonald JJ, Theeuwes J (2006) Electrophysiological evidence of the capture of visual attention. J Cogn Neurosci 18:604-613. CrossRef Medline

Hickey C, Di Lollo V, McDonald JJ (2009) Electrophysiological indices of target and distractor processing in visual search. J Cogn Neurosci 21:760775. CrossRef Medline

Hilimire MR, Hickey C, Corballis PM (2012) Target resolution in visual search involves the direct suppression of distractors: evidence from electrophysiology. Psychophysiology 49:504-509. CrossRef Medline

Hopf JM, Luck SJ, Boelmans K, Schoenfeld MA, Boehler CN, Rieger J, Heinze HJ (2006) The neural site of attention matches the spatial scale of perception. J Neurosci 26:3532-3540. CrossRef Medline

Ipata AE, Gee AL, Goldberg ME, Bisley JW (2006) Activity in the lateral intraparietal area predicts the goal and latency of saccades in a freeviewing visual search task. J Neurosci 26:3656-3661. CrossRef Medline

Jannati A, Gaspar JM, McDonald JJ (2013) Tracking target and distractor processing in fixed-feature visual search: evidence from human electrophysiology. J Exp Psychol Hum Percept Perform 39:1713-1730. CrossRef Medline

Jolicoeur P, Brisson B, Robitaille N (2008) Dissociation of the N2pc and sustained posterior contralateral negativity in a choice response task. Brain Res 1215:160-172. CrossRef Medline

Kiss M, Grubert A, Petersen A, Eimer M (2012) Attentional capture by salient distractors during visual search is determined by temporal task demands. J Cogn Neurosci 24:749-759. CrossRef Medline

Kumada T, Humphreys GW (2002) Cross-dimensional interference and cross-trial inhibition. Percept Psychophys 64:493-503. CrossRef Medline

Lavie N (1995) Perceptual load as a necessary condition for selective attention. J Exp Psychol Hum Percept Perform 21:451-468. CrossRef Medline

Leber AB (2010) Neural predictors of within-subject fluctuations in attentional control. J Neurosci 30:11458-11465. CrossRef Medline

Luck SJ, Hillyard SA (1994a) Electrophysiological correlates of feature analysis during visual search. Psychophysiology 31:291-308. CrossRef Medline

Luck SJ, Hillyard SA (1994b) Spatial filtering during visual search: evidence from human electrophysiology. J Exp Psychol Hum Percept Perform 20: 1000-1014. CrossRef Medline

Luck SJ, Girelli M, McDermott MT, Ford MA (1997a) Bridging the gap between monkey neurophysiology and human perception: an ambiguity resolution theory of visual selective attention. Cogn Psychol 33:64-87. CrossRef Medline

Luck SJ, Chelazzi L, Hillyard SA, Desimone R (1997b) Neural mechanisms of spatial selective attention in areas V1, V2, and V4 of macaque visual cortex. J Neurophysiol 77:24-42. Medline

Mazza V, Turatto M, Umiltà C, Eimer M (2007) Attentional selection and identification of visual objects are reflected by distinct electrophysiological responses. Exp Brain Res 181:531-536. CrossRef Medline

McDonald JJ, Ward LM (1999) Spatial relevance determines facilitatory and inhibitory effects of auditory covert spatial orienting. J Exp Psychol Hum Percept Perform 25:1234-1252. CrossRef

McDonald JJ, Green JJ, Jannati A, Di Lollo V (2013) On the electrophysiological evidence for the capture of visual attention. J Exp Psychol Hum Percept Perform 39:849-860. CrossRef Medline

Moran J, Desimone R (1985) Selective attention gates visual processing in the extrastriate cortex. Science 229:782-784. CrossRef Medline

Mounts JR (2000a) Evidence for suppressive mechanisms in attentional selection: feature singletons produce inhibitory surrounds. Percept Psychophys 62:969-983. CrossRef Medline

Mounts JR (2000b) Attentional capture by abrupt onsets and feature singletons produces inhibitory surrounds. Percept Psychophys 62:1485-1493. CrossRef Medline

Müller HJ, Geyer T, Zehetleitner M, Krummenacher J (2009) Attentional capture by salient color singleton distractors is modulated by top-down dimensional set. J Exp Psychol Hum Percept Perform 35:1-16. CrossRef Medline

Sawaki R, Luck SJ (2010) Capture versus suppression of attention by salient 
singletons: electrophysiological evidence for an automatic attend-to-me signal. Atten Percept Psychophys 72:1455-1470. CrossRef Medline

Sawaki R, Geng JJ, Luck SJ (2012) A common neural mechanism for preventing and terminating the allocation of attention. J Neurosci 32:1072510736. CrossRef Medline

Theeuwes J (1991) Cross-dimensional perceptual selectivity. Percept Psychophys 50:184-193. CrossRef Medline

Theeuwes J (1992) Perceptual selectivity for colour and form. Percept Psychophys 51:599-606. CrossRef Medline

Töllner T, Zehetleitner M, Gramann K, Müller HJ (2011) Stimulus saliency modulates pre-attentive processing speed in human visual cortex. PLoS One 6:e16276. CrossRef Medline

Töllner T, Müller HJ, Zehetleitner M (2012) Top-down dimensional weight set determines the capture of visual attention: evidence from the PCN component. Cereb Cortex 22:1554-1563. CrossRef Medline
Ulrich R, Miller J (2001) Using the jackknife-based scoring method for measuring LRP onset effects in factorial designs. Psychophysiology 38:816827. CrossRef Medline

Vogel EK, Machizawa MG (2004) Neural activity predicts individual differences in visual working memory capacity. Nature 428:748-751. CrossRef Medline

Woodman GF, Luck SJ (2003) Serial deployment of attention during visual search. J Exp Psychol Hum Percept Perform 29:121-138. CrossRef Medline

Wykowska A, Schubö A (2010) On the temporal relation of top-down and bottom-up mechanisms during guidance of attention. J Cogn Neurosci 22:640-654. CrossRef Medline

Wykowska A, Schubö A (2011) Irrelevant singletons in visual search do not capture attention but can produce nonspatial filtering costs. J Cogn Neurosci 23:645-660. CrossRef Medline 